\title{
BIM-ORIENTED ALGORITHMIC RECONSTRUCTION OF BUILDING COMPONENTS FOR EXISTING HERITAGE
}

\author{
C. Giannattasio ${ }^{1, *}$, L. M. Papa ${ }^{1}$, P. D’Agostino ${ }^{1}$ \\ ${ }^{1}$ Department of Civil, Architectural and Environmental Engineering, University of Naples Federico II, Naples, Italy \\ (carlo.giannattasio, lmpapa, pierpaolo.dagostino)@unina.it
}

Commission II, WG II/8

KEY WORDS: EBIM, Heritage, Algorithmic design, Digitization, CADToBIM, ScanToBIM

\begin{abstract}
:
This study is part of a more complex research aimed at establishing guidelines to simplify the digitalization process used to manage existing building heritage. Working in a BIM environment, this paper will present two different algorithms: a modelling algorithm, a data analysis algorithm, and relative applications in the digitalization of a contemporary building. All the archival data required for the digitalization process was collected and those in two-dimensional digital vector format have aroused particular interest because they enabled initiation of the reconstruction process of the BIM model. One of the two algorithms allowed us to identify recurrent elements in a CAD drawing, based on geometric 2D primitives. The final outcome of the first phase involves quadrilateral or circular surfaces and can be viewed in algorithmic environment. The next phase involves applying a unique coloured sign to the identified sections and then export them all in a BIM software. This tool produced unexpected positive results: the presence of a small coloured grid emphasized the discrepancies created between the two-dimensional drawings and the vertical elements. We were thus able to identify the objects with these inconsistencies: they were verified using accurate surveys and then corrected.
\end{abstract}

\section{INTRODUCTION}

\subsection{Scope of the research}

This study is part of a more complex research aimed at establishing guidelines for public administrations, working in a BIM environment to simplify the digitalization process used to manage existing building heritage (Giannattasio et al., 2019).

It focuses on integrating visual scripting tools into the process used to create models for existing buildings, in particular the definition of objects with complex forms and the analysis and discretisation of geometric input data. The paper will also illustrate results regarding the use of two applications in the process used to create the BIM model of a building owned by the University of Naples Federico II.

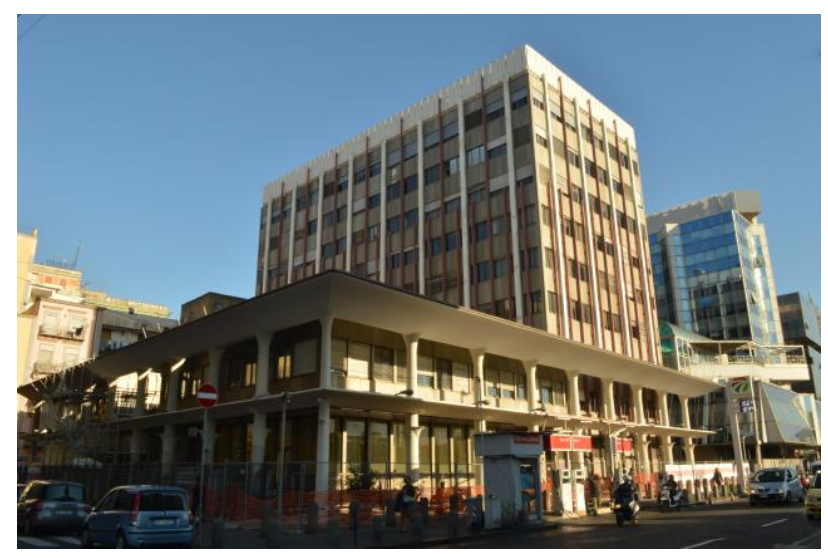

Figure 1. The office building of the University of Naples Federico II

\subsection{BIM and the problems regarding existing buildings}

BIM modelling makes it possible to combine graphic and computational data in a single three-dimensional object; the object summarizes everything in a prototype/clone of the study object (Rosen et al., 2015) containing more information than each graphic and non-graphic image considered individually. As we all know, one of the major advantages is undoubtedly the prospect of having a single model in which different disciplines coexist, for example architectural, structural or engineering disciplines. Other advantages include the possibility to draft more accurate multidimensional analyses and to instantly generate twodimensional drawings and 3D views, thereby reducing the time needed to produce tables and projects. However, these advantages are not limited only to the design and implementation of works: with an appropriately developed model it is possible to optimize the management of a building during its useful life (Bianchini et al., 2017). Management is not limited only to aspects regarding the building (Carbonari et al., 2015), such as systems management, but can also include, for example, the management of personnel, mobile heritage and spaces (Kassem et al., 2015).

Given the potential advantages regarding management, this methodology is increasingly applied to existing building heritage, especially public buildings; in Italy the latter represent approximately $23.08 \%$ (230,536 buildings) of all existing building heritage (998,937 buildings) and cover an area of $292,632,285 \mathrm{~m} 2$, i.e., $85.51 \%$ of the total surface area (Ministero delle Economie e delle Finanze, 2015). One must also consider that existing building heritage includes buildings that date well beyond the 150 years that have passed since the Unification of Italy. Over the years, not only have graphic and textual documents not always been recorded in a reasoned and rational manner, they also vary in their conformation and type,

\footnotetext{
* Corresponding author
} 
thereby complicating standardization, especially as regards a BIM-oriented approach.

Regulatory compliance and maintenance and management requirements are gradually forcing public administrations to use BIM models to update documents based on CAD drawings. However, this process has several intrinsic problems, e.g., assessment of the availability and reliability of the data required to create the model. To solve this problem high definition digital surveys are often integrated to reconstruct points clouds in order to implement the ScanToBIM processes (Tommasi et al., 2016). This is not an economically viable solution, especially because the archival data is enormous. In addition, BIM was developed to be used in the design of new buildings and must therefore be adapted to function for existing buildings; this involves a still imperfect optimization of the software and accurate modelling of several building components. To achieve the latter, algorithms and applications are being combined to facilitate recognition of surfaces and forms (Wang et al., 2015).

\section{ALGORITHMIC MODELING}

\subsection{Introduction to the subject}

The introduction of algorithmic modelling in the most important software platforms has made it possible to establish procedures that increase the options available to operators, especially as regards the creation of complex objects and forms in both purely generative modelling environments (Capone, Lanzara, 2019) and in parametric BIM platforms (Paris, Wahbeh, 2016, di Luggo et al., 2018); the aim is to make different data import and export programs interact (Bonduel et al., 2018). However, these tools do more than this: in fact, wellstructured algorithms can automatize and optimize processes (Evins, 2013) and either generate numerous different solutions or analyze huge amounts of data in a short space of time (Caetano, Leitão, 2018). However, the potential of computational design presents just as many operational difficulties: if on the one hand these tools can solve very complex problems (Salimzadeh et al., 2018), on the other the operator has to have expert knowledge of the discipline involved in order to provide the best possible description of the operations and parameters to be introduced. Furthermore, the expertise required to successfully use these tools presents intrinsic difficulties: these skills are not limited to engineering and architecture but often involve mathematics and computing (Pavan, Mirarchi, 2019). If on the one hand visual scripting tools simplify the process of writing algorithms, on the other a programmer has to be proficient in order to develop complex applications.

This paper will present two different algorithms: a modelling algorithm, a data analysis algorithm, and relative applications in the digitalization of a contemporary building. The selected building is located in Via Giulio Cesare Cortese (Naples); it has a reinforced concrete frame, a curtain wall envelope, and is functionally divided into three parallelepipeds: the biggest has eight floors while the other two floors are symmetrically located along its longer side.

The building, constructed in the seventies, was purchased in 1997 by the University of Naples Federico II and used as offices. Last year the Technical Offices of the university requested a paradigmatic example of a BIM model in order to proceed with the digitalization of all the university's building heritage.

Choosing this building did not depend only on the specific request by the technical offices for an initial prototype. In fact, to understand how it could be updated and integrated with all the necessary information we decided to start with a scenario that contained comprehensive graphic and informative material and then apply a BIM process without having to perform a new digital survey. Archival research showed that there were numerous digital drawings which, albeit with all the abovementioned problems (some of which are described below), were deemed suitable to facilitate digitalization of the building.

\subsection{The analytical algorithm: semi-automatic CADToBIM}

All the archival data required for the digitalization process was collected; the images drafted after a recent survey were of particular interest because they enabled initiation of the reconstruction process of the BIM model. To provide the technical office with a tool to simplify the digitalization of the data in semi-automatic forms we developed an algorithm that identified recurrent elements in a CAD drawing, based on geometric 2D primitives. By treating the initial data as a set of basic geometric shapes we decided to create a repertoire of graphic signs that could act as reference for ordinary or recurrent objects inside a building. With this in mind, the set was limited to quadrangular or circular components; in fact, the latter can represent either structural elements such as pilasters and columns, or elements of the systems, such as pipes and conduits.

The measure of the vectorial entity was another discretionary parameter for recognition: in fact, it is automatically excluded if it does not belong to the interval in question. Having identified the components required and after discarding the others, we initially intended to automatize the input of the BIM objects; however we later abandoned this option, leaving operators a certain degree of discretion when identifying errors and false positives. Automatic positioning was thus replaced by a tagging process which allowed recognition of two-dimensional vectorial elements, differentiated by colour. In fact, by combining the same colour hues with identical parameters, the algorithm simplifies the ensuing input process of the objects by positioning a point in the centre of the section, thereby optimizing the CADToBIM process.

\subsection{Functioning of the analytical algorithm}

After illustrating the result to be achieved we established the input parameters:

- the CAD drawing;

- a minimum and maximum numerical interval;

- the level at which the result is visible.

After establishing the parameters, the process was divided into two macro phases: the first phase involves reducing the overall number of definitions, while during the second phase the elements in question are identified and labelled.

During the first phase all the objects in the CAD drawing were imported into the BIM environment and divided according to their size and solid digital nature. Since this involves quadrangular or circular sections that fall within the specific interval, the application automatically excludes whatever does not represent the element in question (e.g., text boxes, points, grids, etc.) and whatever is either too big or too small, and therefore not within a plausible range for architecturally valid sections for building components.

At this point it is important to differentiate: while the circular sections can be easily identified given their axial-symmetry (useful to automatize the shift to the tapping phase), this was not the case for quadrangular sections. 


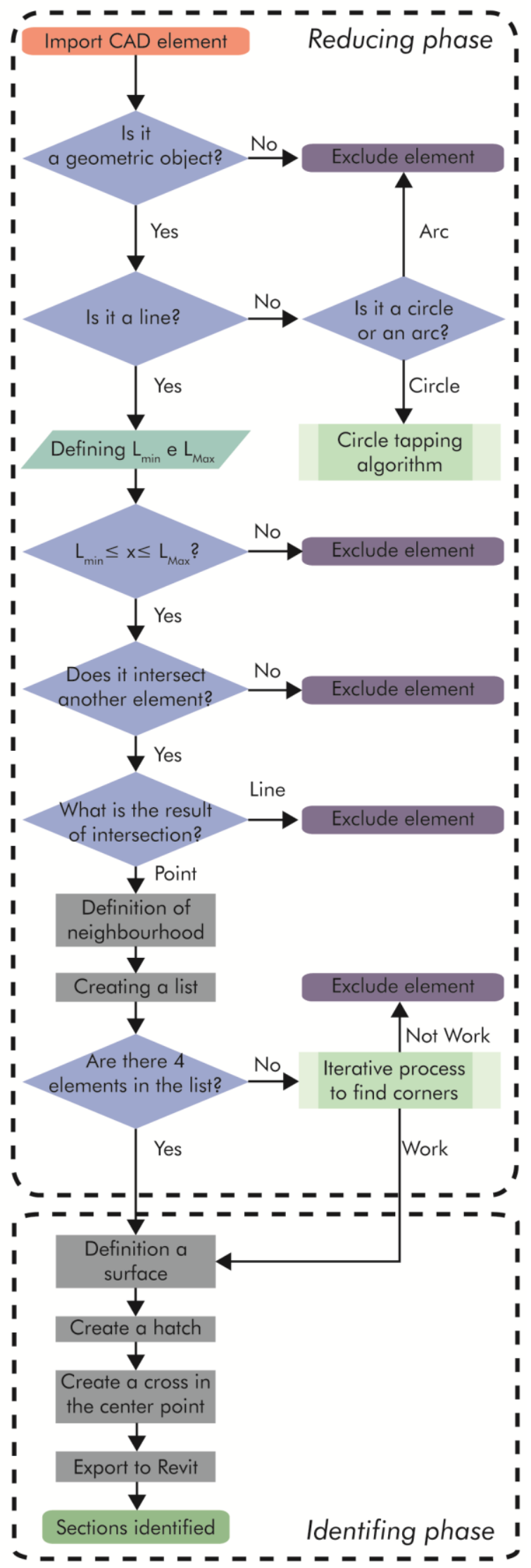

Figure 2. Flowchart of the analytical algorithm for quadrangular sections
The nature of line objects, which obviously make up these sections, required a longer process to discretize the elements in question. In fact the software tool restricted direct recognition of multilinear vectorial elements - widely used to reconstruct the relative closed sections of the structural profiles of the building - and instead provided a breakdown into single "line" objects. So we needed to proceed gradually, but only after excluding the curved objects. All these lines are then identified and intersected so as to exclude the ones that do not intersect (and are therefore not the sides of a quadrilateral) and all double lines. The result of this operation is a set of points which, for each group, represents the vertexes of the same number of quadrilaterals. The ensuing lists contain four components which are then ordered so that it is possible to create a closed surface. Should there be more points, the list is analyzed separately; the aim of this iterative process is to exclude all the points that do not belong to corners. If even then it is impossible to obtain a suitable number of elements, the algorithm excludes the whole group so as to avoid creating errors. The final outcome of this first phase involves quadrilateral or circular surfaces and can be viewed in a Dynamo ${ }^{\oplus}$ environment.

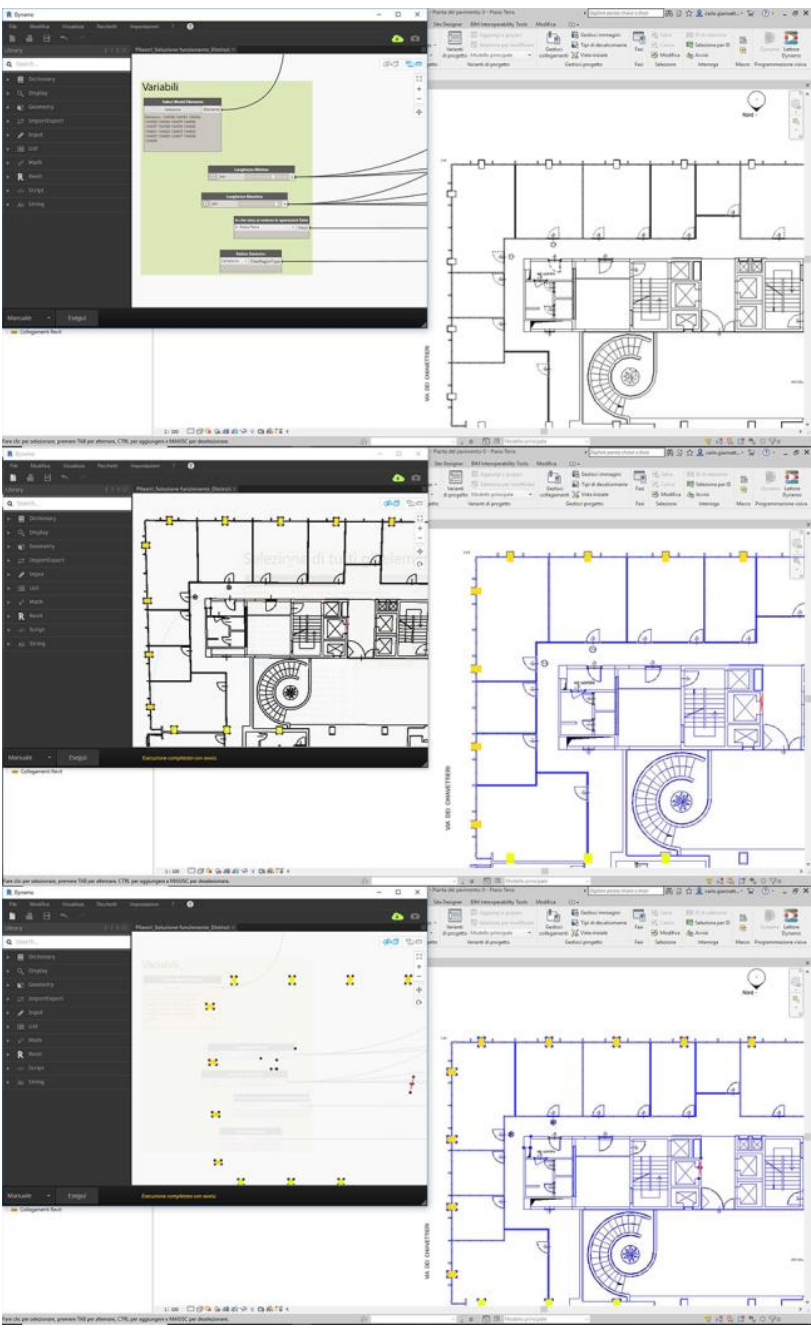

Figure 3. Functioning of the analytical algorithm. On the top, process start. On the centre, processing phase. On the bottom, final phase and results 
The next phase involves applying a unique coloured sign to the identified sections and then export them all in a Revit $\odot$ environment. To do this, we found it useful to hatch these sections with a colour that changed hue depending on the normalized numerical value associated with the area. By making small changes to the algorithm it was possible to develop another application that enabled identification of specific rectangular sections with known long and short sides.
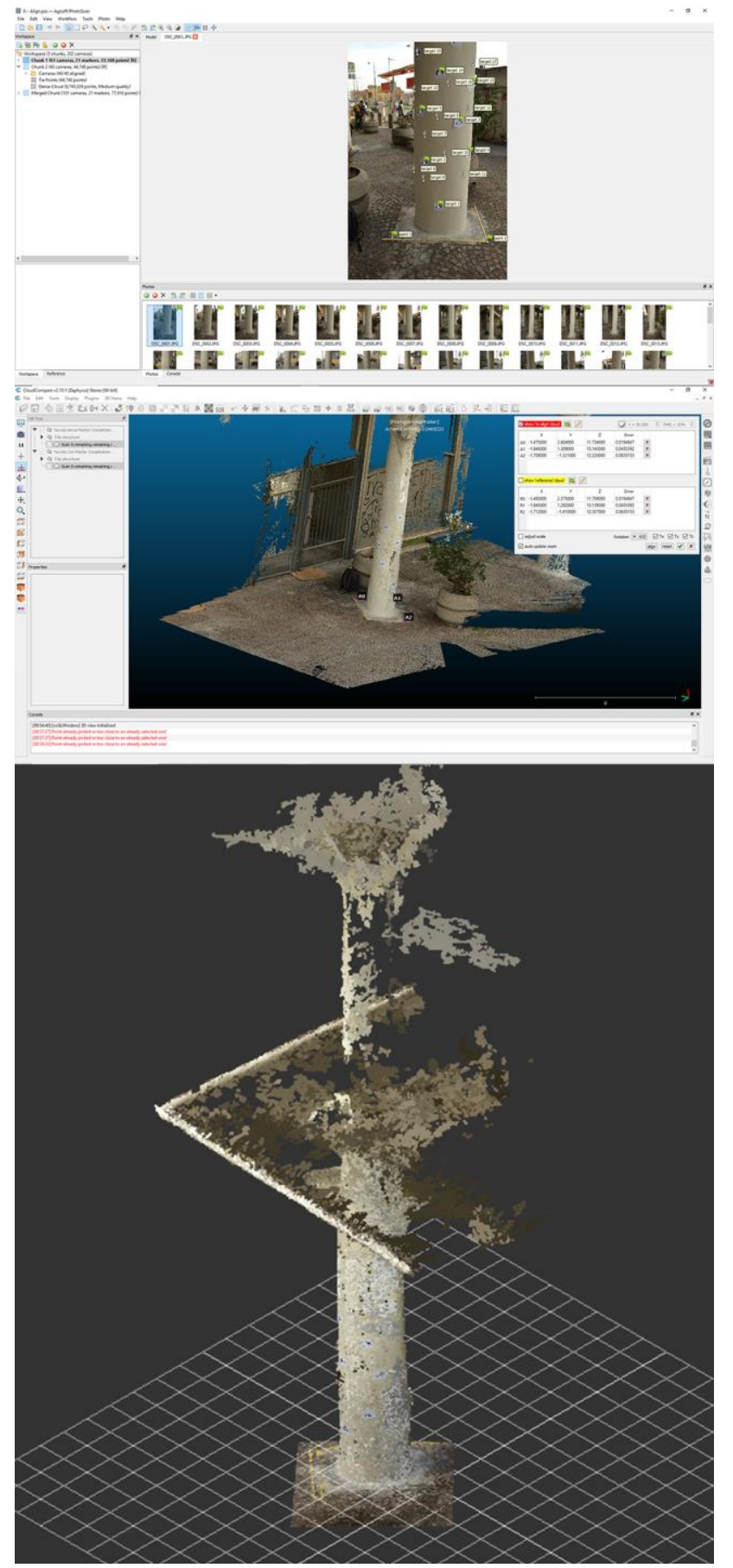

Figure 4. Creating the column point cloud. On the top, reconstruction of the point cloud through digital photogrammetry. On the centre, cleaning phase. On the bottom, final phase and results.

\subsection{The modelling algorithm: the external columns}

A difficulty arose during the reconstruction of the BIM model of the building; it involved the reconstruction of the external colonnade. The only data regarding this component came from the elevations which turned out to be incomplete and therefore insufficient for a three-dimensional reconstruction. In particular, the drawings did not fully portray the complexity of the column which had a continuous but irregular shape and had two skylights at different levels.

As a result we had to perform a digital survey for the restitution of a points cloud generated by a digital stereoscopic photogrammetry process. We had to use a marker-based approach to recreate the surface of the component because it was very reflective and certain aspects of its axial symmetry complicated the reconstruction process.

Once the reconstruction of the points cloud was completed, a ScanToBim method was used to initially try to reconstruct the column in a Revit platform, envisaging the use of specific templates for families of objects; however we were unable to recreate objects that corresponded geometrically to the real object. So we tried to create it as a mass solid. We obtained several significant sections which were then used to create the shaft of the column. The capital was modelled separately from the rest of the shaft since the environment in which the column was created was unable to define a single object with its specific characteristics. So they were modelled separately and then united using Boolean operators. Illuminating objects, shaped in reinforced concrete and integrated into the shaft, were modelled using the same method, i.e., using a workflow similar to the construction of the shaft of the column; they were then united in a similar manner, by applying Boolean operators to the whole complex.

Another difficulty was encountered regarding the definition of the second illuminating object, located higher up compared to the first: having copied and rotated the first, the software was unable to unite the second one with the shaft due to the shape of the model of the shaft. The solution involved integrating the algorithm with a change in the basic reference planes, identified at a specific level; the next step was to proceed with a solid synthesis by uniting the solids. However the latter cannot be turned into a family of columns: this means that the object is not valid from a computational point of view, even thought it is valid geometrically. We decided to link the computational design tool to the family environment so as to create a geometry as similar as possible to the real object. Having previously analyzed the points cloud very scrupulously, it was easy to reconstruct the sequence of the ensuing vertical sections. All that was left to do was to establish a series of operations to create objects or Booleans that would allow the reconstruction of a solid very similar to the real one. The outcome was successful.

\section{RESULTS AND FUTURE DEVELOPMENTS}

The aim of this study was to highlight the potential of visual scripting tools in the reconstruction of BIM models of existing heritage. By integrating the semi-automatic CAD TO BIM procedures it was possible to simplify the input process of prevalently vertical objects, e.g., pilasters or columns on a seamless façade. In addition, tagging produced unexpected, positive results: the presence of a small coloured grid emphasized the discrepancies created between the twodimensional drawings and the vertical elements. We were thus able to identify the objects with these inconsistencies: they were verified using accurate surveys and then corrected. 

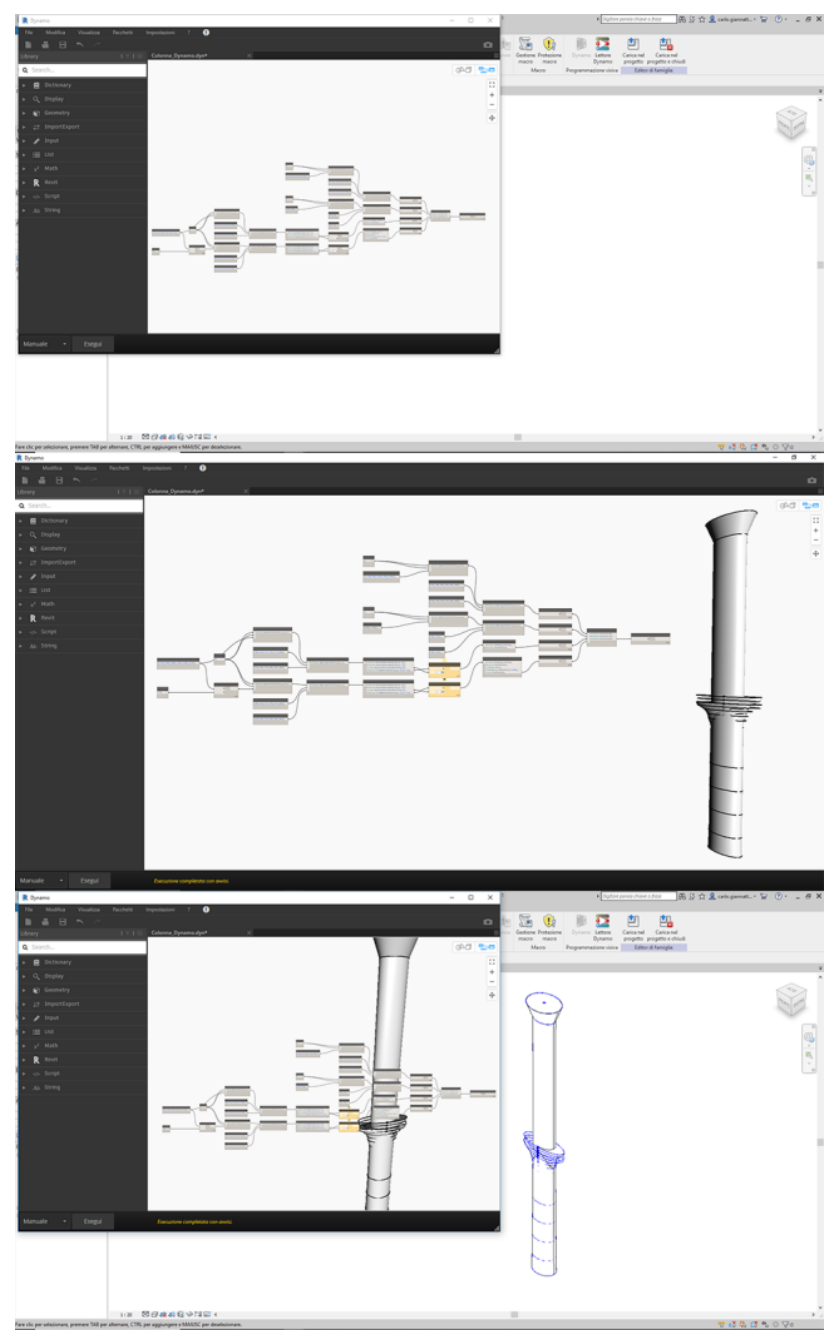

Figure 5. Functioning of the modelling algorithm. On the top, process start. On the centre, processing phase. On the bottom, final phase and results.

Likewise, in some cases algorithmic modelling is the only possible solution, for example to create geometries that are both complex and valid computationally. Studying the column highlighted this unique situation: although it was impossible to reconstruct the element after creating a family, the second attempt in a mass environment enabled the reconstruction of the structure of the object, but not its nature. Emphasis should be placed on the fact that the algorithm used to reconstruct the column - formally complex but ultimately suitably defined as a correct class of objects - represents a specific typification algorithm. In other words it can be used only to reconstruct this type of column and cannot, under any circumstances, be generalized.

However, it is also true that the results of this study will be crucial during the next phases of the research, i.e., to establish procedures and tools for the digitalization and management of building heritage. In order to achieve optimization we will attempt to create new algorithms that simplify the interaction between points clouds and BIM objects.

\section{CONCLUSIONS}

Using BIM models on existing buildings provides searchable and easily updated digital data that can be used to manage said buildings. Reconstruction of these models is not always straightforward, not least because the main BIM platforms are not optimized for this kind of procedure. However the introduction of visual scripting tools has made it possible to increase the number of tools available to technical operators. In fact, algorithmic design broadens the possibility to create complex forms and objects and reduces the time involved in repetitive operations. Nevertheless, not only are specialized operators needed to develop these algorithms, but a detailed study of the specific problems is also required; if satisfactorily defined, the study will make the development of these applications advantageous.

When developing these algorithms special attention must be paid to the detail to be studied: trying to overgeneralize a problem may require too many work hours and thus become an economically invalid solution. At the same time, developing an application to create a single element may make the app unusable in other circumstances. As a result the operator has to assess whether or not the perfect geometrisation of the BIM object is necessary or whether a simplified version suffices, thereby saving time and money during reconstruction of the model. A non-complex geometry may nonetheless be valid if the computational nature of the BIM object is respected. It is therefore crucial for operators to possess computer skills so that they can not only manage this new type of data, but also develop valid, optimized algorithms.

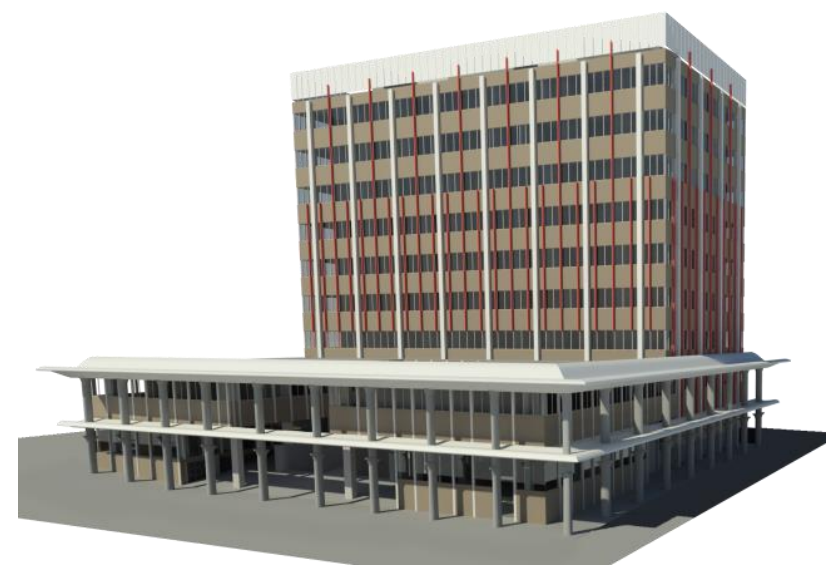

Figure 6. Final rendering of the BIM model

\section{REFERENCES}

Bianchini, C., Inglese, C., Ippolito, A., Maiorino, D., Senatore, L. J., 2017. Building Information Modeling (BIM): Great Misunderstanding or Potential Opportunities for the Design Disciplines?. In: A., Ippolito, M., Cigola, Handbook of Research on Emerging Technologies for Digital Preservation and Information Modeling. IGI Global, Hershey, PA, pp. 67-90.

Bonduel, M., Vergauwen, M., Klein, R., Rasmussen, M.H., Pauwels, P., 2018. A novel workflow to combine BIM and Linked Data for existing buildings. In 11th European Conference on Product and Process Modelling, CRC Press, 347-354.

Caetano, I., Leitão, A., 2018. Integration of an Algorithmic BIM Approach in a Traditional Architecture Studio. Journal of 
Computational Design Engineering, pp.327-336, https://www.doi.org/10.1016/j.jcde.2018.11.004.

Capone, M., Lanzara, E., 2019. Scan-To vs 3d Ideal Model Hbim: Parametric Tools to Study Domes Geometry. ISPRSInternational Archives of the Photogrammetry, Remote Sensing and Spatial Information Sciences, XLII-2/W9, pp. 219-226, https://doi.org/10.5194/isprs-archives-XLII-2-W9-219-2019.

Carbonari, G., Stravoravdis, S., Gausden, C., 2015. Building information model implementation for existing buildings for facilities management: a framework and two case studies. Building Information Modelling (BIM). in Design, Construction and Operations. Southampton, UK: WIT Press, pp. 395-406, https://www.doi.org/10.2495/BIM150331.

di Luggo, A., Scandurra, S., Pulcrano, M., Siconolfi, M., Monaco, S., 2018. Sistemi voltati nei processi cloud to BIM Vaulted systems in cloud to BIM procedures. In $3 D$ MODELING \& BIM - Nuove Frontiere, DEI s.r.l. Tipografia Del Genio Civile, Roma, pp. 182 - 197.

Evins, R., 2013. A review of computational optimisation methods applied to sustainable building design. Renewable sustainable energy reviews; 22, pp. 230-245, https://doi.org/10.1016/j.rser.2013.02.004.

Giannattasio, C., Papa, L. M., D'Agostino, P., 2019. From line to volume: BIM application for university facilities management - De la línea al volumen: el BIM para la gestión de edificios universitarios. In: APEGA 2019 De la línea a la nube. Almería: Editorial circulo rojo, pp. 201-203.

Kassem, M., Kelly, G., Dawood, N., Serginson, M., Lockley, S., 2015. BIM in facilities management applications: a case study of a large university complex. Built Environment Project and Asset Management, 5, pp. 261-277, https://www.doi.org/10.1108/BEPAM-02-2014-0011.

Logothetis, S., Delinasiou, A., Stylianidis, E., 2015. Building information modelling for cultural heritage: a review. ISPRS Annals of the Photogrammetry, Remote Sensing Spatial Information Sciences. II-5/W3, pp. 177-183, https://doi.org/10.5194/isprsannals-II-5-W3-177-2015.

Ministero delle Economie e delle Finanze, 2015. Rapporto sui beni immobili delle Amministrazioni. http://www.dt.tesoro.it/export/sites/sitodt/modules/documenti_it /patrimonio_pubblico/patrimonio_pa/RapportoImmobili_DatiA nno2015.pdf (May 2019).

Paris, L., Wahbeh, W., 2016. Rilievo e rappresentazione delle geometrie parametriche per l'HBIM / Survey and representation of the parametric geometries in HBIM. Disegnarecon, 9, pp. 12.1-12.9.

Pavan, A., Mirarchi, C., 2019. La norma italiana UNI 11337 sulla digitalizzazione del settore costruzioni - UNI 11337: the Italian standard on the digitization of the construction sector, BIM VIEWS Esperienze e Scenari. Quaderni di incontri sul disegno, Coperativa Universitaria Athena, Fisciano, Vol. 12, pp. 11-20.

Rosen, R., von Wichert, G., Lo, G., Bettenhausen, K. D., 2015. About The Importance of Autonomy and Digital Twins for the Future of Manufacturing, IFACPapersOnLine, Vol. 48-3,
Elvesier, pp. 567-

https://doi.org/10.1016/j.ifacol.2015.06.141.

Salimzadeh, N., Vahdatikhaki, F., Hammad, A., 2018. BIMbased surface-specific solar simulation of buildings. In ISARC. Proceedings of the International Symposium on Automation and Robotics in Construction, IAARC Publications, Vol. 35, pp. 18 .

Tommasi, C., Achille, C., Fassi, F., 2016. From point cloud to BIM: a modelling challange in the Cultural Heritage field. International Archives of the Photogrammetry, Remote Sensing and Spatial Information Sciences, XLI-B5, pp. 429-436, doi:10.5194/isprsarchives-XLI-B5-429-2016.

Volk, R., Stengel, J., Schultmann, F., 2014. Building Information Modeling (BIM) for existing buildings - Literature review and future needs. Automation in construction, 38, pp. 109-127, https://doi.org/10.1016/j.autcon.2013.10.023.

Wang, C., Cho, Y. K., Kim, C., 2015. Automatic BIM component extraction from point clouds of existing buildings for sustainability applications. Automation in Construction, 56, pp. 1-13, https://doi.org/10.1016/j.autcon.2015.04.001. 